\title{
Computing pure Nash and strong equilibria in bottleneck congestion games
}

\author{
Tobias Harks • Martin Hoefer • Max Klimm • \\ Alexander Skopalik
}

Received: 19 October 2010 / Accepted: 2 February 2012 / Published online: 8 March 2012 (C) The Author(s) 2012. This article is published with open access at Springerlink.com

\begin{abstract}
Bottleneck congestion games properly model the properties of many realworld network routing applications. They are known to possess strong equilibria-a strengthening of Nash equilibrium to resilience against coalitional deviations. In this paper, we study the computational complexity of pure Nash and strong equilibria in these games. We provide a generic centralized algorithm to compute strong equilibria, which has polynomial running time for many interesting classes of games such as, e.g., matroid or single-commodity bottleneck congestion games. In addition, we examine the more demanding goal to reach equilibria in polynomial time using natural improvement dynamics. Using unilateral improvement dynamics in matroid games pure Nash equilibria can be reached efficiently. In contrast, computing even a single coalitional improvement move in matroid and single-commodity games is strongly NP-hard. In addition, we establish a variety of hardness results and lower bounds regarding the
\end{abstract}

An extended abstract of this paper appeared in the Proceedings of the 18th Annual European Symposium on Algorithms (ESA).

T. Harks $(\varangle)$

School of Business and Economics, Maastricht University, Maastricht, The Netherlands

e-mail: t.harks@maastrichtuniversity.nl

M. Hoefer

Department of Computer Science, RWTH Aachen University, Aachen, Germany

e-mail: mhoefer@cs.rwth-aachen.de

\section{Klimm}

Department of Mathematics, TU Berlin, Berlin, Germany

e-mail: klimm@math.tu-berlin.de

A. Skopalik

TU Dortmund, Dortmund, Germany

e-mail: alexander.skopalik@tu-dortmund.de 
duration of unilateral and coalitional improvement dynamics. They continue to hold even for convergence to approximate equilibria.

Keywords Bottleneck congestion games - Computation of strong equilibria · Improvement dynamics

Mathematics Subject Classification 91A10 Noncooperative games · 91A46 Combinatorial games $\cdot 91-08$ Computational methods $\cdot 90 \mathrm{~B} 18$ Communication networks

\section{Introduction}

One of the central challenges in algorithmic game theory is to characterize the computational complexity of equilibria. Results in this direction yield important indicators if game-theoretic solution concepts are plausible outcomes of competitive environments in practice. Probably the most prominent stability concept in (non-cooperative) game theory is the Nash equilibrium - a state, from which no player wants to unilaterally deviate - and its complexity has been under increased scrutiny for quite some time. A drawback of Nash equilibrium is that in general it exists only in mixed strategies. There are, however, practically important classes of games that allow pure Nash equilibria (PNE), most prominently congestion games. In a congestion game [41], there is a set of resources, and the pure strategies of players are subsets of this set. Each resource has a delay function depending on the load, i.e., the number of players that select strategies containing the respective resource. The individual cost for a player in a ordinary congestion game is given by the sum over the delays of the resources in his strategy.

Congestion games are an elegant model to study the effects of resource usage and congestion with strategic agents. They have been used frequently to model competitive network routing scenarios [42]. For these games the complexity of exact and approximate PNE is now well-understood. A detailed characterization in terms of, e.g., the structure of strategy spaces $[2,18]$ or the delay functions $[8,11,45]$ has been derived. However, ordinary congestion games have shortcomings, especially as models for the prominent application of routing in computer networks. The throughput of a stream of packets is usually determined by the delay experienced due to available bandwidth or capacity of links. Here the throughput of a player is closely related to the performance of the most congested (bottleneck) link (see, e.g., [6,12,31,40]). A model that captures this aspect more realistically are bottleneck congestion games, in which the individual cost of a player is the maximum (instead of sum) of the delays in his strategy. Despite being a more realistic model for network routing, they have not received similar attention in the literature. For classes of non-atomic (with infinitesimally small players) and atomic splittable games (finite number of players with arbitrarily splittable demand) existence of PNE and bounds on the price of anarchy were considered in [12,36]. For atomic games with unsplittable demand PNE do always exist [6]. In fact, Harks et al. [25] establish the finite improvement property via a lexicographic potential function. Interestingly, they are able to extend these conditions to hold even if coalitions of players are allowed to change their strategy in a coordinated way. This implies that bottleneck congestion games do admit even (pure) strong equilibria (SE), 
a solution concept introduced by Aumann [5]. In an SE, no coalition (of any size) can deviate and strictly decrease the individual cost of each member. Every SE is a PNE, but the converse holds only in special cases (e.g., for singleton games [28]).

SE represent a very robust and appealing stability concept. In general games, however, they are quite rare, which makes the existence guarantee in bottleneck congestion games even more remarkable. For instance, even in dominant strategy games such as the Prisoner's Dilemma there might be no SE. Not surprisingly, for ordinary congestion games with linear aggregation the existence of SE is not guaranteed $[28,32]$ and, in fact, NP-hard to decide [27]. The existence of PNE and SE in bottleneck congestion games raises a variety of important questions regarding their computational complexity. In which cases can PNE and SE be computed efficiently? As the games have the finite improvement property, another important issue is the duration of natural (coalitional) improvement dynamics. More fundamentally, it is not obvious that even a single such coalitional improving move can be found efficiently. These are the main questions that we address in this paper.

\subsection{Our results}

We examine the computational complexity of PNE and SE in bottleneck congestion games. In Sect. 2 we focus on computing PNE and SE using (centralized) algorithms. Our first main result is a generic algorithm that computes an SE for any bottleneck congestion game. The algorithm iteratively decreases capacities on the resources and relies on a strategy packing oracle. The oracle decides if a given set of capacities allows to pack a collection of feasible strategies for all players and outputs a feasible packing if one exists. The running time of the algorithm is essentially determined by the running time of this oracle, i.e., the problem of computing SE can be reduced to solving the strategy packing problem. As a characterization we also prove the reverse direction: The class of set packing problems addressed by strategy packing oracles can be solved efficiently if we can efficiently compute SE in bottleneck congestion games. A slight drawback is that the games constructed in this reduction exhibit a slightly different combinatorial structure than the packing problem. For the case of two players we can circumvent this problem and show polynomial equivalence between packing and SE computation even when we fix the underlying combinatorial structure.

In terms of complexity, we prove a number of upper and lower bounds for specific classes of games. For upper bounds we focus on three classes of games: single-commodity networks, branchings, and matroids (see Sect. 2.2 for the definition of single-commodity networks and branchings and Sect. 1.3 for the definition of matroids). Single-commodity network games represent a natural and frequently studied class of network routing. Branchings model a natural scenario when players strive to implement a broadcast from a set of source nodes to all other nodes in the network. Finally, matroid games have been studied prominently in ordinary congestion games [2] and represent a straightforward extension of the popular singleton case. In all these cases, there are strategy packing oracles that can be implemented in polynomial time. Thus, our generic algorithm yields an efficient algorithm to compute SE for all these classes of games. For general games, however, we show that the problem of computing an SE is NP-hard, even in two-commodity networks. 
In Sect. 3 we study the duration and complexity of sequential improvement dynamics that converge to PNE and SE. Note that quick convergence (i.e., in a polynomial number of rounds) implies efficient computation. Therefore, we focus particularly on the classes of games, for which we found positive results in terms of computation. In particular, we first observe that for every matroid bottleneck congestion game a variant of best response dynamics presented in [2] called "lazy best response" converges in polynomial time to a PNE. In contrast to this positive result for unilateral dynamics, we show that it is NP-hard to decide if a coalitional improving move exists, even for matroid and single-commodity network games, and even if the deviating coalition is fixed a priori. This highlights an interesting contrast for these two classes of games: While there are polynomial-time algorithms to compute an SE, it is impossible to decide efficiently if a given state is an SE-the decision problem is co-NP-hard.

For more general games, we observe in Sect. 3.2 that constructions of [45] regarding the hardness of computing PNE in ordinary games can be adjusted to yield similar results for bottleneck games. In particular, in (a) symmetric games with arbitrary delay functions and (b) asymmetric games with bounded-jump delay functions computing a PNE is PLS-complete. In addition, we show that in both cases there exist games and starting states, from which every sequence of improvement moves to a PNE is exponentially long. We extend this result to the case when moves of coalitions of size $\mathcal{O}\left(n^{1-\epsilon}\right)$ are allowed, for any constant $\epsilon>0$. In addition, we observe that all of these hardness results generalize to the computation of $\alpha$-approximate PNE and SE (see Sect. 1.3 for the definition), for any polynomially bounded factor $\alpha$.

We conclude the paper in Sect. 4 by outlining some interesting open problems regarding the convergence to approximate equilibria.

\subsection{Related work}

Congestion games (in the ordinary sense) were introduced by Rosenthal [41] and further characterized by Monderer and Shapley [38]. Holzman and Law-Yone [28] studied the existence of SE in congestion games with monotone increasing delay functions. They showed that SE need not exist in such games and gave a structural characterization of the strategy space for symmetric (and quasi-symmetric) congestion games that admit SE. They also introduced the concept of a strong potential function: a function on the set of states that decreases for every profitable deviation of a coalition. More recently, Hoefer and Skopalik [27] showed that deciding existence of SE in congestion games is NP-hard, even for two players, and even in games which are both matroid and single-commodity network games. Rozenfeld and Tennenholtz [43] explored the existence of (correlated) SE in congestion games with non-increasing delay functions. Exact and approximate SE have also been considered in other games, e.g., in cost sharing congestion games $[3,17,34]$.

A generalization of congestion games has been proposed by Milchtaich [37], where he allows for player-specific delay functions on the resources (see also [1,24,27,35] for subsequent work on (weighted) congestion games with player-specific delay functions). For games with singleton strategies and monotonic delay functions, Milchtaich proves existence of PNE. As shown by Voorneveld et al. [47], the singleton games 
considered by Milchtaich are equivalent to the games considered by Konishi et al. [32]. This is worth noting as they established existence of SE in such games. Closely related, Andelman et al. [4] considered scheduling games on unrelated machines and proved that the load-lexicographically minimal schedule is an SE. Efficiency of strong equilibria in scheduling games has been studied by Fiat et al. [21], and notions of approximate strong equilibria were analyzed by Feldman and Tamir [19].

Bottleneck congestion games with network structure have been considered by Banner and Orda [6]. They proved existence of PNE in the unsplittable and splittable flow settings. Harks et al. [25] considered a generalization of bottleneck congestion games and proved that these games possess the strong finite improvement property. Epstein et al. [16] characterized network topologies for both ordinary and bottleneck network congestion games such that in the resulting games all PNE are socially optimal. The price of anarchy for PNE in bottleneck congestion games was studied in $[9,14,30]$.

Bottleneck routing with non-atomic players and elastic demands has been studied by Cole et al. [12], who derived bounds on the price of anarchy. For subsequent work on the price of anarchy in bottleneck routing games with atomic and non-atomic players, we refer to the paper by Mazalov et al. [36].

\subsection{Preliminaries}

Bottleneck congestion games are strategic games $G=\left(N, \mathcal{S},\left(c_{i}\right)_{i \in N}\right)$, where $N=$ $\{1, \ldots, n\}$ is the non-empty and finite set of players, $\mathcal{S}=\chi_{i \in N} \mathcal{S}_{i}$ is the non-empty set of states or strategy profiles, and $c_{i}: \mathcal{S} \rightarrow \mathbb{N}$ is the individual cost function that specifies the cost value of player $i$ for each state $S \in \mathcal{S}$. A game is called finite if $\mathcal{S}$ is finite. For the sake of a clean mathematical definition, we define strategies and costs using the general notion of a congestion model. A tuple $\mathcal{M}=\left(N, R, \mathcal{S},\left(d_{r}\right)_{r \in R}\right)$ is called a congestion model if $N=\{1, \ldots, n\}$ is a non-empty, finite set of players, $R=\{1, \ldots, m\}$ is a non-empty, finite set of resources, and $\mathcal{S}=\chi_{i \in N} \mathcal{S}_{i}$ is the set of states or profiles. For each player $i \in N$, the set $\mathcal{S}_{i}$ is a non-empty, finite set of pure strategies $S_{i} \subseteq R$. Given a state $S$, we define $\ell_{r}(S)=\left|\left\{i \in N: r \in S_{i}\right\}\right|$ as the number of players using $r$ in $S$. Every resource $r \in R$ has a delay function $d_{r}: S \rightarrow \mathbb{N}$ defined as $d_{r}(S)=d_{r}\left(\ell_{r}(S)\right)$. In this paper, all delay functions are non-negative and non-decreasing. Delay function $d_{r}$ satisfies the $\beta$-bounded-jump condition if $d_{r}(x+1) \leq \beta \cdot d_{r}(x)$ for any $x \geq 1$. A congestion model $\mathcal{M}$ is called matroid congestion model if for every $i \in N$ there is a matroid $M_{i}=\left(R, \mathcal{I}_{i}\right)$ such that $\mathcal{S}_{i}$ equals the set of bases of $M_{i}$. We denote by $\operatorname{rk}(\mathcal{M})=\max _{i \in N} \operatorname{rk}\left(M_{i}\right)$ the rank of the matroid congestion model. (Bottleneck) congestion games corresponding to matroid congestion models will be called matroid (bottleneck) congestion games. Matroids exhibit numerous nice properties, some of which are summarized in the Appendix A.1. For a comprehensive overview see standard textbooks [33, Chapter 13] and [44, Chapters 39-42].

Let $\mathcal{M}$ be a congestion model. The corresponding bottleneck congestion game is the strategic game $G(\mathcal{M})=\left(N, \mathcal{S},\left(c_{i}\right)_{i \in N}\right)$ in which $c_{i}$ is given by $c_{i}(S)=$ $\max _{r \in S_{i}} d_{r}\left(\ell_{r}(S)\right)$. We drop $\mathcal{M}$ whenever it is clear from context. We define the corresponding ordinary congestion game in the same way, the only difference is that 
$c_{i}(S)=\sum_{r \in S_{i}} d_{r}\left(\ell_{r}(S)\right)$. For a coalition $C \subseteq N$ we denote by $-C$ its complement and by $\mathcal{S}_{C}=\chi_{i \in C} \mathcal{S}_{i}$ the set of states of players in $C$. A pair $\left(S,\left(S_{C}^{\prime}, S_{-C}\right)\right) \in \mathcal{S} \times \mathcal{S}$ is called an $\alpha$-improving move of coalition $C$ if $c_{i}(S)>\alpha c_{i}\left(S_{C}^{\prime}, S_{-C}\right)$ for all $i \in C$ and $\alpha \geq 1$. For $\alpha=1$ we call $\left(S,\left(S_{C}^{\prime}, S_{-C}\right)\right)$ an improving move (or profitable deviation). A state $S$ is a $k$-strong equilibrium $(k-\mathrm{SE})$, if there is no improving move $(S, \cdot)$ for a coalition of size at most $k$. We say $S$ is a strong equilibrium (SE) if and only if it is an $n$-SE. Similarly, $S$ is a pure Nash equilibrium (PNE) if and only if it is a 1-SE. We call a state $S$ an $\alpha$-approximate SE (PNE) if no coalition (single player) has an $\alpha$-improving move $(S, \cdot)$. We denote by $I(S)$ the set of all possible improving moves $\left(S, S^{\prime}\right)$ to other states $S^{\prime} \in \mathcal{S}$. We call a sequence of states $\left(S^{0}, S^{1}, \ldots\right)$ an improvement path if every tuple $\left(S^{k}, S^{k+1}\right) \in I\left(S^{k}\right)$ for all $k=0,1,2, \ldots$ Intuitively, an improvement path is a path in a so-called state graph $\mathcal{G}(G)$ derived from $G$, where every state $S \in \mathcal{S}$ corresponds to a node in $\mathcal{G}(G)$ and there is a directed edge $\left(S, S^{\prime}\right)$ if and only if $\left(S, S^{\prime}\right) \in I(S)$.

\section{Computing strong equilibria}

In this section, we investigate the complexity of computing an SE in bottleneck congestion games. We first present a generic algorithm that computes an SE for an arbitrary bottleneck congestion game. It uses an oracle that solves a strategy packing problem (see Definition 1), which we term strategy packing oracle. For games in which the strategy packing oracle can be implemented in polynomial time, we obtain an efficient algorithm computing an SE. We then examine games for which this is the case. In general, however, we prove that computing an SE is NP-hard, even for two-commodity bottleneck congestion games.

\subsection{The dual greedy}

The general approach of our algorithm is to introduce upper bounds $u_{r}$ (capacities) on each resource $r$. The idea is to iteratively reduce upper bounds of costly resources as long as the residual capacities admit a feasible strategy packing, see Definition 1 below. Our algorithm can be interpreted as a dual greedy, or worst out algorithm as studied, e.g., in the field of network optimization, see Schrijver [44].

\section{Definition 1 (Strategy packing oracle)}

INPUT: Finite set of resources $R$ with upper bounds $\left(u_{r}\right)_{r \in R}$, and $n$ collections $\mathcal{S}_{1}, \ldots, \mathcal{S}_{n} \subseteq 2^{R}$ given implicitly by a certain combinatorial property.

OUTPUT: Sets $S_{1} \in \mathcal{S}_{1}, \ldots, S_{n} \in \mathcal{S}_{n}$ such that $\left|i \in\{1, \ldots, n\}: r \in S_{i}\right| \leq u_{r}$ for all $r \in R$, or the information, that no such sets exist.

More specifically, when the algorithm starts, no strategy has been assigned to any player and each resource can be used by $n$ players, thus, $u_{r}=n$. If $r$ is used by $n$ players, its delay equals $d_{r}(n)$. The algorithm now iteratively reduces the maximum resource delay by picking a resource $r^{\prime}$ with maximum delay $d_{r}\left(u_{r}\right)$ and $u_{r}>0$. The number of players allowed on $r^{\prime}$ is reduced by one and the strategy packing oracle checks, if there is a feasible strategy profile obeying the capacity constraints. If 


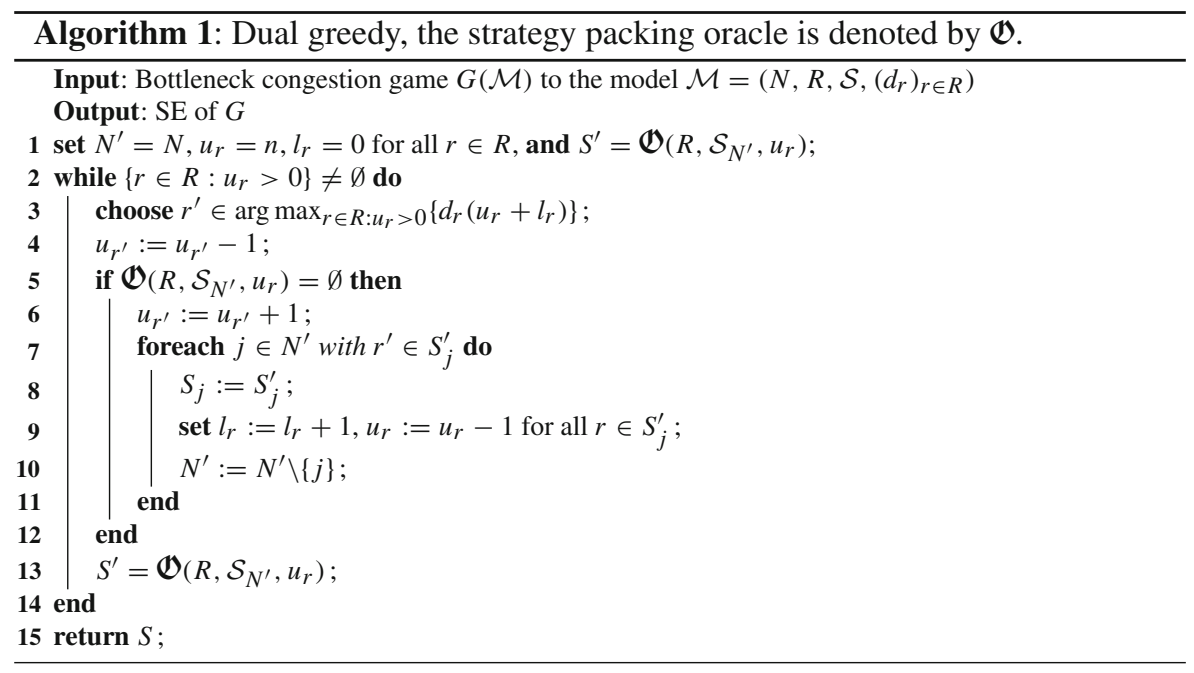

the strategy packing oracle outputs such a feasible state $S$, the algorithm reiterates by choosing a (possibly different) resource that has currently maximum delay. If the strategy packing oracle returns $\emptyset$ after the capacity of some $r^{\prime} \in R$ was reduced to $u_{r^{\prime}}-1$, we fix the strategies of those $u_{r^{\prime}}$ many players that used $r^{\prime}$ in the state the strategy packing oracle computed in the previous iteration and decrease the bounds $u_{r}$ of all resources used in the strategies accordingly. This ensures that $r^{\prime}$ is frozen, i.e., there is no residual capacity on $r^{\prime}$ for allocating this resource in future iterations of the algorithm. The algorithm terminates after at most $n \cdot m$ calls of the oracle. For a formal description of the algorithm see Algorithm 1.

Theorem 1 Dual greedy computes an SE.

Proof Let $S$ denote the output of the algorithm. In addition, we denote by $N_{k}, k=$ $1, \ldots, K$, the sets of players whose strategies are determined after the strategy packing oracle (denoted by $\mathfrak{U}$ ) returned $\emptyset$ for the $k$ th time. Clearly, $c_{i}(S) \leq c_{j}(S)$ for all $i \in N_{k}, j \in N_{l}$, with $k \geq l$. We will show by complete induction over $k$ that the players in $N_{1} \cup \cdots \cup N_{k}$ will not participate in any improving move of any coalition.

We start with the case $k=1$. Let $\left(u_{r}\right)_{r \in R}$ be the vector of capacities in the algorithm after the strategy packing oracle returned $\emptyset$ in line 5 for the first time and $u_{r^{\prime}}$ is updated in line 6.

Suppose there is a coalition $C \subseteq N$ with $C \cap N_{1} \neq \emptyset$ that deviates profitably from $S$ to $T=\left(S_{C}^{\prime}, S_{-C}\right)$. We distinguish two cases.

Case 1: $\ell_{r}(T) \leq u_{r}$ for all $r \in R$. Let $\tilde{u}_{r}=u_{r}-1$, if $r=r^{\prime}$ and $\tilde{u}_{r}=u_{r}$, else. Since $\mathfrak{G}(R, \mathcal{S}, \tilde{u})=\emptyset$, at least $\left|N_{1}\right|$ players use $r^{\prime}$ in $T$. Using $d_{r^{\prime}}(T) \geq d_{r}(S)$ for all $r \in R$, we obtain a contradiction to the fact that every member of $C$ must strictly improve.

Case 2: There is $\tilde{r} \in R$ such that $\ell_{\tilde{r}}(T)>u_{\tilde{r}}$. Using that dual greedy iteratively reduces the capacity of those resources with maximum delay (line 3 ), we derive that 
$d_{\tilde{r}}(T) \geq d_{r}(S)$ for all $r \in R$. Using $\ell_{\tilde{r}}(T)>u_{\tilde{r}}$ and $\ell_{r}(S) \leq u_{r}$ for all $r \in R$, there is at least one player $i \in C$ with $\tilde{r} \in S_{i}^{\prime}$, hence, this player does not strictly improve.

For the induction step $k \rightarrow k+1$, suppose the players in $N_{1} \cup \cdots \cup N_{k}$ stick to their strategies and consider the players in $N_{k+1}$. As the strategies of the players in $N_{1} \cup \cdots \cup N_{k}$ are fixed, the same arguments as above imply that no subset of $N_{k+1}$ will participate in a profitable deviation from $S$.

It is worth noting that the dual greedy algorithm applies to arbitrary strategy spaces. If the strategy packing problem can be solved in polynomial time, this algorithm computes an SE in polynomial time. This follows easily because the algorithm calls the packing oracle at most $n \cdot m$ times.

Corollary 1 For bottleneck congestion games in which the strategy packing problem is polynomial-time solvable, dual greedy computes an SE in polynomial time.

While the problem of computing an SE is polynomial-time reducible to the strategy packing problem, for general bottleneck congestion games the converse is also true.

Theorem 2 The strategy packing problem is polynomial-time reducible to the problem of computing an SE in a bottleneck congestion game.

Proof Given an instance of the strategy packing problem $\Pi$ we construct a bottleneck congestion game $G_{\Pi}$. Let $\Pi$ be given as set of resources $R$ with upper bounds $\left(u_{r}\right)_{r \in R}$, and $n$ collections $\mathcal{S}_{1}, \ldots, \mathcal{S}_{n} \subseteq 2^{R}$. The game $G_{\Pi}$ consists of the resources $R \cup\left\{r_{1}, \ldots, r_{n}\right\}$ and the players $1, \ldots, n+1$. The set of strategies of player $i \in$ $\{1, \ldots, n\}$ is $\left\{S_{i} \cup\left\{r_{i}\right\} \mid S_{i} \in \mathcal{S}_{i}\right\}$. Player $n+1$ has the strategies $R$ and $\left\{r_{1}, \ldots, r_{n}\right\}$. For each resource $r \in R$ the delay is 0 if used by at most $u_{r}+1$ players and 2 otherwise. For each resource $r \in\left\{r_{1}, \ldots, r_{n}\right\}$ the delay is 0 if used by at most one player and 1 otherwise.

If a strategy profile of players $1, \ldots, n$ violates an upper bound $u_{r}$ on a resource $r \in R$, player $n+1$ has delay of 2 if he plays strategy $R$. If he plays $\left\{r_{1}, \ldots, r_{n}\right\}$, he and all other players have delay of 1 . Hence, if there is a feasible strategy packing, every $\mathrm{SE}$ of the game yields delay 0 for every player. Otherwise, every SE yields delay 1 for every player. Therefore, the state of the players $1, \ldots, n$ in an SE of $G_{\Pi}$ corresponds to a solution for the strategy packing problem $\Pi$, if such a solution exists. On the other hand, if there is no solution for $\Pi$, every player in every SE in $G_{\Pi}$ has delay of 1 .

Note that while the previous theorem establishes a reduction in general, the game $G_{\Pi}$ constructed from the instance $\Pi$ of the packing problem has a different combinatorial structure than $\Pi$. More concretely, $G_{\Pi}$ is based on a larger set of resources and different strategy sets than the ones used in $\Pi$. The next theorem shows that for games with two players, we can obtain a stronger equivalence result without changing the underlying combinatorial structure. It remains an open problem to extend this stronger result to games with an arbitrary number of players and more general packing problems.

Theorem 3 Let $R$ be a finite set and $\mathcal{S}_{1}, \mathcal{S}_{2} \subseteq 2^{R}$ two collections of subsets of $R$. Then the following two problems are polynomially equivalent: 
1. Compute an $S E$ of $G(\mathcal{M})$ for the congestion model $\mathcal{M}=\left(\{1,2\}, R, \mathcal{S},\left(d_{r}\right)_{r \in R}\right)$ where $\left(d_{r}\right)_{r \in R}$ is an arbitrary set of non-decreasing delay functions.

2. Compute $S_{1} \in \mathcal{S}_{1}, S_{2} \in \mathcal{S}_{2}$ such that $\left.\mid i \in\{1,2\}: r \in S_{i}\right\} \mid \leq u_{r}$ or decide that no such strategies exist where $u_{r} \in\{1,2\}$ for all $r \in R$ is arbitrary.

Proof "2. $\rightarrow$ 1.": As the dual greedy algorithm computes an SE using polynomial many calls of the strategy packing oracle the first problem is polynomially reducible to the second one.

"1. $\rightarrow$ 2.": Suppose we are given an instance $\left(R, \mathcal{S},\left(u_{r}\right)_{r \in R}\right)$ of the second problem. We regard the congestion model $\mathcal{M}=\left(\{1,2\}, R, \mathcal{S},\left(d_{r}\right)_{r \in R}\right)$ where $d_{r}$ is defined as

$$
d_{r}(\ell)= \begin{cases}0, & \text { if } \ell \leq u_{r} \\ 1, & \text { otherwise }\end{cases}
$$

Now, let $G$ be a corresponding bottleneck congestion game and let $S^{*}$ be an SE of $G$. We claim that $c_{1}\left(S^{*}\right)=c_{2}\left(S^{*}\right)=0$ and $S^{*}$ is a solution of the strategy packing problem if such a solution exists, and $c_{1}\left(S^{*}\right)=c_{2}\left(S^{*}\right)=1$, otherwise. At first, note that $u_{r} \in\{1,2\}$, and therefore a player gets a delay of 1 if and only if there is $r \in S_{1}^{*} \cap S_{2}^{*}$ with $u_{r}=1$. In this case, however, both players have a delay of 1 , so we have either $c_{1}\left(S^{*}\right)=c_{2}\left(S^{*}\right)=1$ or $c_{1}\left(S^{*}\right)=c_{2}\left(S^{*}\right)=0$. Suppose that $c_{1}\left(S^{*}\right)=c_{2}\left(S^{*}\right)=1$ and assume for a contradiction that there is solution $S^{\prime}=\left(S_{1}^{\prime}, S_{2}^{\prime}\right)$ to the strategy packing problem. Then, by the definition of $d_{r}$ we get that $c_{1}\left(S^{\prime}\right)=c_{2}\left(S^{\prime}\right)=0$ and, thus, the deviation from $S^{*}$ to $S^{\prime}$ is profitable both for player 1 and 2. This is a contradiction to the fact that $S^{*}$ is an SE. Hence, no such state $S^{\prime}$ exists.

For the other direction, it is easy to check that $c_{1}\left(S^{*}\right)=c_{2}\left(S^{*}\right)=0$ only if the strategies $S_{1}^{*}$ and $S_{2}^{*}$ obey the upper bounds on each resource.

\subsection{Complexity of strategy packing}

In the previous section we have characterized the computation of SE in terms of a set packing problem. In this section, we examine the computational complexity of strategy packing and SE computation. In particular, we consider three classes of games, in which strategy packing can be done efficiently. For the general case, we show that computation becomes NP-hard. A more detailed characterization as to which structural properties are crucial for efficient strategy packing or hardness is an interesting avenue for future work.

Our first result is for matroid games, which represent a natural extension of singleton games. In a singleton game we have $\left|S_{i}\right|=1$ for every strategy $S_{i} \in \mathcal{S}_{i}$ of every player $i$. In such games, SE are exactly the PNE and computation of SE can trivially be done in polynomial time. Also, strategy packing reduces to perfect matching in a bipartite graph. ${ }^{1}$ For matroid games, we have to resort to more advanced algorithmic techniques.

\footnotetext{
1 We construct the graph as follows. The first partition contains a node for each player, the second partition contains $u_{r}$ nodes for each $r \in R$. The node of player $i$ is connected to all nodes of each $r \in \mathcal{S}_{i}$.
} 
Theorem 4 The strategy packing problem can be solved in polynomial time for matroid bottleneck congestion games where the strategy set of player i equals the set of bases of a matroid $M_{i}=\left(R, \mathcal{I}_{i}\right)$ given by a polynomial independence oracle.

Proof For each matroid $M_{i}=\left(R, \mathcal{I}_{i}\right)$, we construct a matroid $M_{i}^{\prime}=\left(R^{\prime}, \mathcal{I}_{i}^{\prime}\right)$ as follows. For each resource $r \in R$, we introduce $u_{r}$ resources $r^{1}, \ldots, r^{u_{r}}$ to $R^{\prime}$. We say that $r$ is the representative of $r^{1}, \ldots, r^{u_{r}}$. Then, a set $I^{\prime} \subset R^{\prime}$ is independent in $M_{i}^{\prime}$ if the set $I$ that arises from $I^{\prime}$ by replacing resources by their representatives is independent in $M_{i}$. This construction gives rise to a polynomial independence oracle for $M_{i}^{\prime}$.

Now, we regard the matroid union $M^{\prime}=M_{1}^{\prime} \vee \cdots \vee M_{n}^{\prime}$, see Definition 2 in the Appendix A.1, which again is a matroid. Using the algorithm proposed by Cunningham [13] we can compute a maximum-size set $\mathcal{B}$ in $I_{1}^{\prime} \vee \cdots \vee I_{n}^{\prime}$ in time polynomial in $n, m, r k(\mathcal{M})$, and the maximum complexity of the $n$ independence oracles.

Clearly, if $|\mathcal{B}|<\sum_{i \in N} \operatorname{rk}\left(M_{i}\right)$, there is no feasible packing of the bases of $M_{1}, \ldots, M_{n}$. If, in contrast, $|\mathcal{B}|=\sum_{i \in N} \operatorname{rk}\left(M_{i}\right)$, we obtain the corresponding strategies $\left(S_{1}, \ldots, S_{n}\right)$ using the algorithm.

Let us now consider strategy spaces defined as a-arborescences, which are in general not matroids. Let $\mathcal{D}=(V, R)$ be a directed graph with $|R|=m$. For a distinguished node in $a \in V$, we define an a-arborescence as a directed spanning tree, where $a$ has in-degree zero and every other vertex has in-degree one. In this case, we can regard $a \in V$ as a common source, and each player strives to make a broadcast with source $a$ by allocating a tree.

Theorem 5 The strategy packing problem can be solved in time $\mathcal{O}\left(m^{2} n^{2}\right)$ for aarborescence games in which the set of strategies of each player equals the set of a-arborescences in a directed graph $\mathcal{D}=(V, R)$.

Proof The problem of finding $k$ disjoint $a$-arborescences in $G$ can be solved in polynomial time $\mathcal{O}\left(m^{2} k^{2}\right)$, see Gabow [23, Theorem 3.1]. Introducing $u_{r}$ copies for each edge $r \in R$, the problem of finding admissible strategies in the original problem is equivalent to finding $n$ disjoint $a$-arborescences.

Recently, the polynomial packing algorithm for $a$-arborescences has been extended to branchings. Formally, we are given for each player $i$ a root set $R_{i} \subseteq V$ and a convex ${ }^{2}$ set $U_{i} \subseteq V$ with $R_{i} \subseteq U_{i}$. For any vector of capacities $\left(u_{r}\right)_{r \in R}$, the polynomial algorithm of Bérczi and Frank [7] computes for every player a branching which is rooted in $R_{i}$ and spans $U_{i}$, that is, the in-degree of every vertex $v \in R_{i}$ is zero and the in-degree of every vertex $v \in U_{i} \backslash R_{i}$ is one, such that the capacity restriction of every edge is satisfied. This more general framework allows to model situations in which the players wish to broadcast from multiple broadcasting stations, where the broadcasts need not cover all vertices. It is worth mentioning that the convexity of $U_{i}$ is necessary for efficient computation, because otherwise, the corresponding decision problem turns out to be NP-complete.

\footnotetext{
${ }^{2}$ In this context, a subset of vertices $U \subseteq V$ is called convex if there is no vertex $v \in V \backslash U$ such that there is both a directed path from $v$ to some vertex $u \in U$ and a directed path from some node $u^{\prime} \in U$ to $v$.
} 
When we turn to single-commodity networks, then efficient computation of an SE is possible using well-known flow algorithms to implement the oracle. For more general cases with two commodities, however, a variety of problems concerning SE become NP-hard by a simple construction.

Theorem 6 The strategy packing problem can be solved in time $\mathcal{O}\left(\mathrm{m}^{3}\right)$ for singlecommodity bottleneck congestion games.

Proof Assigning a capacity of $u_{r}$ to each edge and using the algorithm of Edmonds and Karp we obtain a maximum flow within $\mathcal{O}\left(\mathrm{m}^{3}\right)$. Clearly, if the value of the flow is smaller than $n$, no admissible strategies exist and we can return $\emptyset$. If the flow is $n$ or larger we can decompose it in at least $n$ unit flows and return $n$ of them.

Theorem 7 In two-commodity network bottleneck games it is strongly NP-hard to (1) compute an SE, (2) decide for a given state whether any coalition has an improving move, and (3) decide for a given state and a given coalition if it has an improving move.

Proof We reduce from the 2 DIRECTED ARC-DiSJOINT PATHS (2DADP) problem, which is strongly NP-hard, see Fortune et al. [22]. The problem is to decide if for a given directed graph $\mathcal{D}=(V, A)$ and two node pairs $\left(s_{1}, t_{1}\right),\left(s_{2}, t_{2}\right)$ there exist two arc-disjoint $\left(s_{1}, t_{1}\right)$ - and $\left(s_{2}, t_{2}\right)$-paths. For the reduction, we define a corresponding two-commodity bottleneck game by introducing non-decreasing delay functions on every arc $r$ by $d_{r}(x)=0$, if $x \leq 1$ and 1 , else. We associate every commodity with a player. For proving (1), we observe that 2DADP is a Yes-instance if and only if every SE provides a payoff of zero to every player. For proving (2) and (3), we simply construct a solution in which the strategies for both players are not arc-disjoint.

\section{Convergence of improvement dynamics}

In the previous section, we have outlined some prominent classes of games, for which SE can be computed in polynomial time. Furthermore, it is known [25] that sequential improvement dynamics converge to PNE and SE. In this section, we consider the duration of improvement dynamics in these games. As polynomial-time convergence implies polynomial-time computation, we first focus on classes of games, in which we have shown efficient computation, i.e., matroid and single-commodity network games. For matroid games we show polynomial-time convergence to a PNE using unilateral improving moves. For the convergence to SE we have to consider coalitional improving moves, but we show that deciding if such a move exists is NP-hard even in matroid games or single-commodity network games. This implies that even in these specialized classes of games with efficient computation of an SE, recognition of a state as an SE is co-NP-hard.

In more general games, hardness of recognition is not the only source of difficulty. In particular, we prove that in general games even computing an $\alpha$-approximate PNE is PLS-hard. There are games and starting states, for which every sequence of unilateral improving moves is exponentially long. Perhaps surprisingly, this also holds when we consider coalitional improving moves of coalitions of size $O\left(n^{1-\epsilon}\right)$, for any constant $\epsilon>0$. 


\subsection{Matroid and single-commodity network games}

We first observe that bottleneck congestion games can be transformed into ordinary congestion games while preserving useful properties regarding the convergence to PNE. This allows to show fast convergence to PNE in matroid bottleneck games and mirrors a prominent result for ordinary matroid games [2].

\subsubsection{Convergence to pure Nash equilibria}

The following lemma establishes a connection between bottleneck and ordinary congestion games. For a bottleneck congestion game $G$ we denote by $G^{\mathrm{sum}}$ the ordinary congestion game with the same congestion model as $G$ except that we choose $d_{r}^{\prime}(S)=$ $m^{d_{r}(\cdot)}, r \in R$.

Lemma 1 Every PNE for $G^{\text {SUm }}$ is a PNE for $G$.

Proof Suppose $S$ is a PNE for $G^{\text {Sum }}$ but not for $G$. Thus, there is player $i \in N$ and strategy $S_{i}^{\prime} \in \mathcal{S}_{i}$, such that $\max _{r \in S_{i}} d_{r}\left(\ell_{r}(S)\right)>\max _{r \in S_{i}^{\prime}} d_{r}\left(\ell_{r}\left(S_{i}^{\prime}, S_{-i}\right)\right)$. We define $\bar{d}:=\max _{r \in S_{i}^{\prime}} d_{r}\left(\ell_{r}\left(S_{i}^{\prime}, S_{-i}\right)\right)$. This implies $\max _{r \in S_{i}} d_{r}\left(\ell_{r}(S)\right) \geq \bar{d}+1$. We obtain a contradiction by observing

$$
\sum_{r \in S_{i}} d_{r}^{\prime}\left(\ell_{r}(S)\right) \geq \max _{r \in S_{i}} d_{r}^{\prime}\left(\ell_{r}(S)\right) \geq m^{\bar{d}+1}>(m-1) m^{\bar{d}} \geq \sum_{r \in S_{i}^{\prime}} d_{r}^{\prime}\left(\ell_{r}\left(S_{i}^{\prime}, S_{-i}\right)\right) .
$$

We analyze the lazy best response dynamics considered for ordinary matroid congestion games presented in [2]. Note that in matroid games, a player always picks as strategy a basis of a matroid. A lazy best response means that a player only exchanges a minimum number of resources that is needed to arrive at a basis representing a best response strategy (for details see [2]). Our analysis here is quite simple and does not explicitly rely on these details. In particular, we transform the game to an ordinary game as outlined in Lemma 1. Then we use the lazy best response dynamics in the ordinary game and the convergence result of [2] as a "black box" with the slight adjustment that we only execute moves yielding a strict improvement in the bottleneck resource of the moving player. This allows to establish the following result.

Theorem 8 Let $G$ be a matroid bottleneck congestion game. Then the lazy best response dynamics converges to a PNE in at most $n^{2} \cdot m \cdot r k(\mathcal{M})$ steps.

Proof We consider the lazy best response dynamics in the corresponding game $G^{\mathrm{sum}}$. In addition, we suppose that a player accepts a deviation only if his bottleneck value is strictly reduced. This might lead to even earlier termination of the dynamics. Thus, the duration is still bounded from above by $n^{2} \cdot m \cdot \operatorname{rk}(\mathcal{M})$ moves as shown in [2]. 


\subsubsection{Convergence to strong equilibria}

For matroid bottleneck congestion games we have shown above that there are polynomially long sequences of unilateral improving moves to a PNE from every starting state. While previous work [25] also establishes convergence to SE for every sequence of coalitional improving moves, it may already be hard to find one such move. In fact, we show that even an $\alpha$-improving move can be strongly NP-hard to find, for any polynomial-time computable $\alpha$, even if strategy spaces have simple matroid structures. This implies that deciding whether a given state is an $\alpha$-approximate SE is strongly co-NPhard-even if all delay functions satisfy the $\beta$-bounded-jump condition, for any $\beta>\alpha$.

Theorem 9 In matroid bottleneck congestion games it is strongly NP-hard to decide for a given state $S$ if there is some coalition $C \subseteq N$ that has an $\alpha$-improving move, for every polynomial-time computable $\alpha$.

Proof We reduce from SET PACKING. An instance of SET PACKING is given by a set of elements $E$ and a set $\mathcal{U}$ of sets $U \subseteq E$, and a number $k$. The goal is to decide if there are $k$ mutually disjoint sets in $\mathcal{U}$. Given an instance of SET PACKING we show how to construct a matroid game $G$ and a state $S$ such that there is an improving move for some coalition of players $C$ if and only if the instance of SET PACKING has a solution.

The game will include $|N|=1+|\mathcal{U}|+|E|+\sum_{U \in \mathcal{U}}|U|$ many players. First, we introduce a master player $p_{1}$, which has two possible strategies. He can either pick a coordination resource $r_{c}$ or the trigger resource $r_{t}$. For each set $U \in \mathcal{U}$, there is a set player $p_{U}$. Player $p_{U}$ can choose either $r_{t}$ or a set resource $r_{U}$. For each set $U$ and each element $e \in U$, there is an inclusion player $p_{U, e}$. Player $p_{U, e}$ can use either the set resource $r_{U}$ or an element resource $r_{e}$. Finally, for each element $e$, there is an element player $p_{e}$ that has strategies $\left\{r_{c}, r_{e}\right\}$ and $\left\{r_{c}, r_{a}\right\}$ for some absorbing resource $r_{a}$.

The state $S$ is given as follows. Player $p_{1}$ is on $r_{c}$, all set players use $r_{t}$, all inclusion players the corresponding set resources $r_{U}$, and all element players the strategies $\left\{r_{c}, r_{e}\right\}$. The coordination resource $r_{c}$ is a bottleneck for the master player and all element players. The delays are $d_{r_{c}}(x)=\alpha+1$, if $x>|E|$ and 1 , otherwise. The trigger resource has delay $d_{r_{t}}(x)=1$, if $x \leq|\mathcal{U}|-k+1$, and $\alpha+1$, otherwise. For the set resources $r_{U}$ the delay is $d_{r_{U}}(x)=1$, if $x \leq 1$ and $\alpha+1$, otherwise. Finally, for the element resources the delay is $d_{r_{e}}(x)=1$ if $x \leq 1$ and $\alpha+1$ otherwise.

Suppose that the underlying SET PACKING instance is a Yes-instance, then an $\alpha$-improving move is as follows. The master player moves to $r_{t}$, the $k$ set players corresponding to a solution choose their set resources, the respective inclusion players move to the element resources, and all element players move to $r_{a}$. The delay of $r_{c}$ reduces from $\alpha+1$ to 1 , and the delay of $r_{t}$ reduces from $\alpha+1$ to 1 . Thus, the master player, all set players, and all element players improve their bottleneck by a factor of $\alpha+1$. The migrating inclusion players do not interfere with each other on the element resources. Thus, they also improve the delay of their bottleneck resource by factor $\alpha+1$, and we have constructed an $\alpha$-improving move for the coalition of all migrating players, all set players, and all element players.

Suppose that the underlying SET PACKING instance is a No-instance. For contradiction, assume that there is a coalition $C$ that has an $\alpha$-improving move. Consider any player $p \in C$. We will show that for any player $p \neq p_{1}$, i.e., any set, inclusion, or 
element player, $p_{1} \in C$ is a prerequisite for achieving any strict improvement. We first note that the master player can never strictly improve without changing his strategy, because all element players will always use $r_{c}$ in their strategy. A move from $r_{c}$ to $r_{t}$ is an improvement if and only if at least $k$ set players drop $r_{t}$. These players must switch to the corresponding resources. However, for a set player $p_{M}$ such a move is an improvement if and only if all inclusion players on $r_{U}$ drop this resource from their strategy. These inclusion players must switch to the element resources. An inclusion player $p_{U, e}$ improves by such a move if and only if the element player drops the resource and $p_{U, e}$ is the only inclusion player moving to $r_{e}$. This implies that the moving set players must correspond to sets that are mutually disjoint. Finally, the element players move from $r_{e}$ to $r_{a}$ with delay $d_{r_{a}}=0$, and this is an improvement if and only if the master player moves away from $r_{c}$. This last argument establishes that $p \in C$ implies $p_{1} \in C$.

However, if the master player $p_{1} \in C$, then we again follow the chain of reasoning above and see that the players corresponding to at least $k$ mutually disjoint sets must move and therefore be in $C$. This is a contradiction to having a No-instance.

Finally, we can add the resource $r_{a}$ to every strategy of the master, set, and inclusion players. In this way, the combinatorial structure of all strategy spaces is the same-a partition matroid $M$ with $r k(M)=2$ and partitions of size 1 and 2-only the mapping to resources is different for each player.

The previous theorem shows hardness of the problem of finding a suitable coalition and a corresponding improving move. Even if we specify the coalition in advance and search only for strategies corresponding to an improving move, the problem remains strongly NP-hard.

Corollary 2 In matroid bottleneck congestion games it is strongly NP-hard to decide for a given state $S$ and a given coalition $C \subseteq N$ if there is an $\alpha$-improving move for $C$, for every polynomial-time computable $\alpha$.

Proof We will show this corollary using the games constructed in the previous proof by fixing the coalition $C=N$. Consider the construction in the previous proof. The coalition described above that has an improving move for a Yes-instance consists of the master player, all set players, all element players and the inclusion players that correspond to the sets of the solution to SET PACKING. However, the inclusion players are only needed to transfer the chain of dependencies to the element players. We can set the strategy space of player $p_{U, e}$ to $\left\{r_{h}, r_{l}\right\} \times\left\{r_{U}, r_{e}\right\}$. Here $r_{h}$ and $r_{l}$ are two resources with delays $d_{r_{h}}=\alpha+1$ and $d_{r_{l}}=0$. In $S$ we assign the inclusion players to strategies $\left\{r_{h}, r_{U}\right\}$. Then an improving move for the inclusion players that remain on $r_{U}$ is to exchange $r_{h}$ by $r_{l}$. Thus, the problem of finding an arbitrary coalition with an improving move becomes trivial. However, we strive to obtain an improving move for $C=N$, and this must generate improvements for the master player and the set players. Thus, we still must reassign some inclusion players from the resources $r_{U}$ to the element resources $r_{e}$. Here we need to resolve conflicts as before, because otherwise inclusion players end up with a delay of $\alpha+1$ on $r_{e}$ and do not improve. Following the previous reasoning we have an $\alpha$-improving move if and only if the underlying SET PACKING instance is solvable. Finally, by appropriately adding dummy resources, we can again ensure that the combinatorial structure of all strategy spaces is the same. 


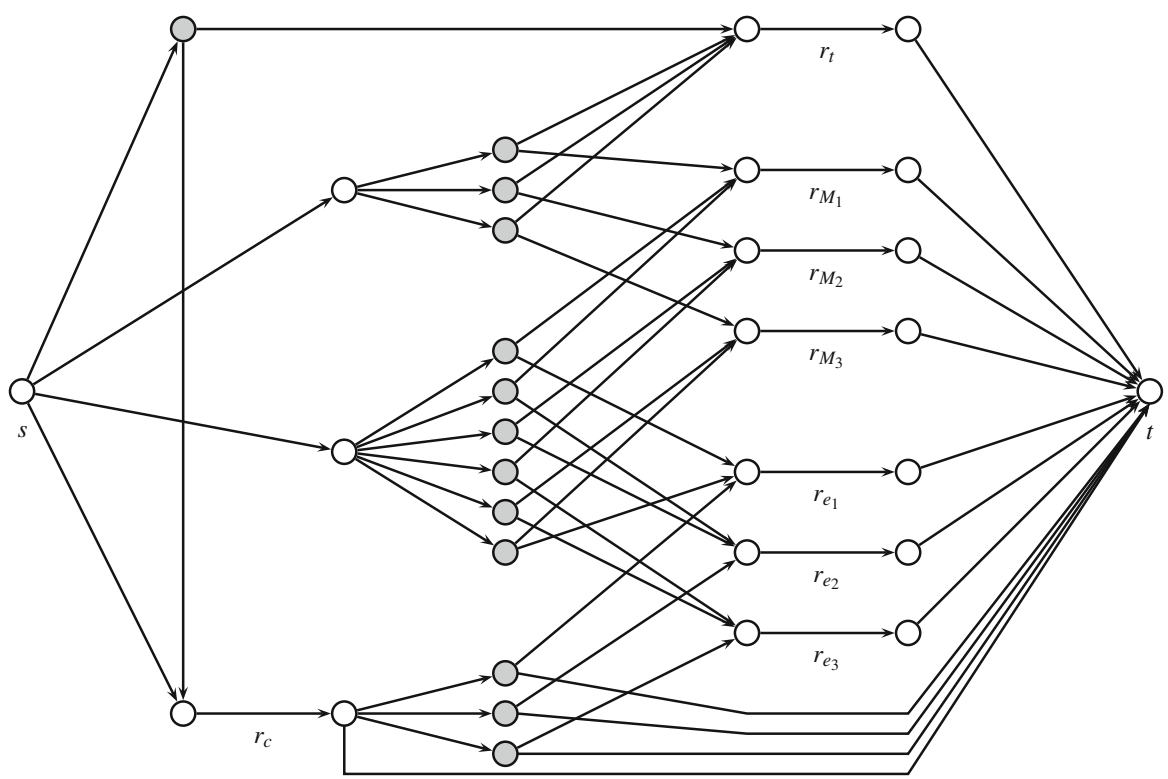

Fig. 1 Network construction for a SET PACKING instance with $\mathcal{U}=\left\{\left\{e_{1}, e_{2}\right\},\left\{e_{2}, e_{3}\right\},\left\{e_{3}, e_{1}\right\}\right\}$. Gray nodes serve as identification for players as discussed in the text

We can adjust the previous two hardness results on matroid games to hold also for single-commodity network games.

Theorem 10 In single-commodity network bottleneck congestion games it is strongly NP-hard to decide for a given state $S$ (1) if there is some coalition $C \subseteq N$ that has an $\alpha$-improving move, and (2) if a given coalition $C \subseteq N$ has an $\alpha$-improving move, for every polynomial-time computable $\alpha$.

Proof We transform the construction of Theorem 9 into a symmetric network bottleneck congestion game, see Fig. 1 for an example. First, we introduce for each resource $r_{c}, r_{t}, r_{U}$ for all $U \in \mathcal{U}$ and $r_{e}$ for all $e \in E$ an edge with the corresponding delay function as before. Additionally, we identify players and their strategies by routing them through a set of gadgets composed of edges, which have capacities implemented by delay functions that are 1 up to a capacity bound and $\alpha+10$ above.

The first gadget is to separate the players into groups. An edge with capacity 1 identifies the master player, an edge with capacity $|\mathcal{U}|$ the set players, an edge with capacity $\sum_{U \in \mathcal{U}}|U|$ the inclusion players, and an edge with capacity $|E|$ the element players. The set and inclusion players are then further divided into their particular identities by edges of capacity 1 . The element players route all over $r_{c}$. In addition, the master player has the alternative to route over $r_{c}$ or $r_{t}$. After the players have passed $r_{c}$ they again split into specific element players using edges of capacity 1 . One player is allowed to route directly to the source $t$. This is meant to be the master player, but it does not hurt our argument if this is not the case. 
After the players have routed through the capacitated gadgets, they can be assumed to reach an identification point (indicated by gray nodes in Fig. 1) and obtain an identity. Then they decide on a strategy from the previous game by routing over one of two allowed paths. In particular, we can allow the set players to route either over $r_{t}$ or their $r_{U}$, the inclusion players over $r_{U}$ or $r_{e}$, and the element players over $r_{e}$ or directly to the sink $t$.

We can create the corresponding state $S$ as before by assigning the master player to route over $r_{c}$ directly to the sink, the set players over $r_{t}$, the inclusion players over $r_{U}$ and the element players over $r_{e}$. This assignment is such that every player receives one identity (i.e., routes over exactly one gray node) and every identity is taken (i.e., every gray node is reached by exactly one player). This property also holds for every improving move-with the exception of one element player, who might route directly from $r_{c}$ to the sink, but as noted before this does not hurt the argument.

Our network structure allows to reconstruct the reasoning as before. Any improving move must include the master player, which improves if and only if he moves together with players corresponding to a solution to the Set Packing instance. Note that even by switching player identities, we cannot create an improving move when the underlying Set Packing instance is unsolvable. This proves the first part of the theorem.

For the second part, we use the same adjustment as in Corollary 2 to ensure that inclusion players can always improve. Directly before the middle fan out (see Fig. 1) that results in identification of inclusion players we simply insert a small gadget with 2 parallel edges $r_{l}$ and $r_{h}$. In this way, all inclusion players must route over one of $r_{l}$ or $r_{h}$ and one of their corresponding $r_{U}$ or $r_{e}$. This resembles the strategy choices in the matroid game and yields hardness of computing an improving move for the coalition $C=N$. This proves the theorem.

\subsection{General games and approximation}

The results of the previous sections imply hardness of the computation of SE or coalitional deviations, even in network games. Therefore, when considering general games we here restrict ourselves mostly to unilateral improving moves and PNE. Unfortunately, even in this restricted case the hardness results for ordinary congestion games in Skopalik and Vöcking [45] immediately imply identical results for bottleneck congestion games. The main result of [45] shows that computing an approximate PNE is PLS-hard. The proof is a reduction from CIRCUITFLIP, a prominent PLS-complete problem for feedback-free Boolean circuits. The problem is to find a local optimum, i.e., a bit string $x$ such that the output resulting from applying the circuit to $x$ cannot be improved lexicographically by switching a single bit in $x$ [29].

We can regard the resulting congestion game in the reduction of [45] as a bottleneck congestion game. It is straightforward to adjust all arguments in the proof of [45] to remain valid for bottleneck congestion games. This simple fact has been observed before, e.g., in [46], and we include it here for completeness. A standard transformation [18] immediately yields the same result even for symmetric games, in which $\mathcal{S}_{i}=\mathcal{S}_{j}$ for all $i, j \in N$. 
Corollary 3 Finding an $\alpha$-approximate PNE in a symmetric bottleneck congestion game with positive and increasing delay functions is PLS-complete, for every polynomial-time computable $\alpha>1$.

A second result in [45] reveals that sequences of $\alpha$-improving moves do not reach an $\alpha$-approximate PNE quickly-even if all delay functions satisfy the $\beta$-bounded-jump condition with a constant $\beta$. Again, the proof remains valid if one regards the game as an asymmetric bottleneck congestion game. This yields the following corollary.

Corollary 4 For every $\alpha>2$, there is a $\beta>1$ such that, for every $n \in \mathbb{N}$, there is a bottleneck congestion game $G(n)$ and a state $S$ with the following properties. The description length of $G(n)$ is polynomial in $n$. The length of every sequence of $\alpha$-improving moves leading from $S$ to an $\alpha$-approximate equilibrium is exponential in $n$. All delay functions of $G(n)$ satisfy the $\beta$-bounded-jump condition.

Using the same trick as before to convert an asymmetric game in a symmetric one yields a similar result for symmetric games. However, we must sacrifice the $\beta$ bounded-jump condition of the delay functions, for every $\beta$ polynomial in $n$.

Despite the fact that (coalitional) improving moves are NP-hard to compute, one might hope that the state graph becomes sufficiently dense such that it allows short improvement paths. Unfortunately, we can show that this is not true, even if we consider all improving moves of coalitions of size up to $\mathcal{O}\left(n^{1-\epsilon}\right)$, for any constant $\epsilon>0$. Again, the same result holds for symmetric games when sacrificing the bounded-jump condition.

Theorem 11 For every $\alpha>2$, there is a $\beta>1$ such that, for every $n \in \mathbb{N}$ and for every $k \in \mathbb{N}$, there is a bottleneck congestion game $G(n, k)$ and a state $S^{\prime}$ with the following properties. The description length of $G(n, k)$ is polynomial in $n$ and $k$. The length of every sequence of $\alpha$-improving moves of coalitions of size at most $k$ leading from $S^{\prime}$ to an $\alpha$-approximate $k$-SE is exponential in $n$. All delay functions of $G(n, k)$ satisfy the $\beta$-bounded-jump condition.

Proof Our proof adjusts the construction of [45], which we recapitulate in the Appendix A.2. The main idea of our adjustment is to construct a bottleneck congestion game $G(n, k)$ by generating $k$ copies of the game $G(n)$. We add resources to the strategies. These resources make sure that there is an improvement step for a player in $G(n)$ if and only if there is an improvement step of corresponding $k$ players of the $k$ copies in $G(n, k)$. For each $t \in\{1, \ldots, 9\}, i \in\{1, \ldots, n\}, m \in\{1, \ldots, k\}$, and $m^{\prime} \neq m$, we add

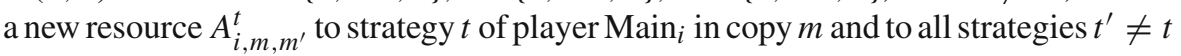
of players Main $_{i}$ of copy $m^{\prime}$. For each $j \in\{1, \ldots, 8\}, i \in\{1, \ldots, n\}, m \in\{1, \ldots, k\}$, and $m^{\prime} \neq m$ we add a new resource $B_{i, m, m^{\prime}}^{j}$ to strategy 1 of player Block ${ }_{i}^{j}$ of copy $m$ and to strategy 2 of Block ${ }_{i}^{j}$ of copy $m^{\prime}$. Each of the above resources added has delay of $\delta^{i-1}$ if it is allocated by at most one player and $\delta^{i+3}$, otherwise.

We obtain the initial strategy profile $S^{\prime}$ of $G(n, k)$ if every player of every copy $m$ of $G(n)$ plays according to the initial strategy profile $S$ of his copy. It it easy to see that no coalition of less than $k$ players has an incentive to change their strategies. At least one of them would have to allocate an $A$ - or $B$-resource that is already in use by a player of 
another copy. Thus, it is not an improvement step for these players. We conclude that all $k$ copies of a player always choose the same strategy. On the other hand, if there is an improving move of one player in $G(n)$, there is a coalitional improving move of all $k$ copies of that player in $G(n, k)$. If all players mimic this deviation in their copies, by construction, no two players allocate the same $A$ - or $B$-resource. Furthermore, if the improvement step decreases the delay in $G(n)$, it does so for every copy of the player in $G(n, k)$.

Finally, note that as long as $k$ is polynomial in $n$ we obtain a reduction of polynomial size. In particular, for $k=n^{1 / \epsilon-1}$ we obtain a new game with $n k$ players, for which the unilateral moves of $G(n)$ are exactly moves of coalitions of size $(n k)^{1-\epsilon}$ and no smaller coalitions have improving moves. This proves the theorem.

\section{Conclusion}

We have provided a detailed study of the computational complexity of exact and approximate pure Nash and strong equilibria in bottleneck congestion games. However, some important and fascinating open problems remain. A major open problem is to find other interesting classes of games, for which efficient computation of and/or fast convergence to SE can be shown. As computation postulates less stringent requirements in terms of locality, there is generally more hope to derive positive results. In particular, what can be said about efficient computation of $\alpha$-approximate SE?

For convergence to SE, we have provided a series of quite strong lower bounds. In this case, it natural to consider weaker concepts of stability that avoid our hardness results. For instance, we did not succeed in translating positive results known for ordinary congestion games and convergence to approximate PNE [8,10,11]. In addition, there are open problems regarding the duration of unilateral dynamics in symmetric network games and hardness of computing PNE in asymmetric networks. Finally, it is a major open problem how to augment the concept of PNE with resilience to coalitional deviations and avoid the hardness results we have observed. It would be interesting to consider computation and convergence characteristics of, e.g., $k$-SE, for $1<k<n$, or equilibrium notions based on player partitions [20] or social networks [26].

Open Access This article is distributed under the terms of the Creative Commons Attribution License which permits any use, distribution, and reproduction in any medium, provided the original author(s) and the source are credited.

\section{Appendix}

Appendix A: Basics in matroid theory

In the following, we will briefly introduce the notion of matroids. For a comprehensive introduction as well as for the proofs of the mentioned results we refer the reader to the textbooks of Korte and Vygen [33, Chapter 13] and Schrijver [44, Chapters 39-42].

Let $F$ be a finite set. A tuple $M=(F, \mathcal{I})$ where $\mathcal{I} \subset 2^{F}$ is called a matroid if (i) $\emptyset \in \mathcal{I}$, (ii) if $I \in \mathcal{I}$ and $J \subseteq I$, then $J \in \mathcal{I}$, and (iii) if $I, J \in \mathcal{I}$ and $|J|<|I|$, then 
there exists an $i \in I \backslash J$ with $J \cup\{i\} \in \mathcal{I}$. A set $A \subseteq F$ is called independent if $A \in \mathcal{I}$ and dependent, otherwise. The set of (inclusion wise) maximal independent subsets of $F$ is called the basis of $M$.

For given $F$, a matroid $(F, \mathcal{I})$ may be of exponential size, thus, one frequently assumes that a matroid comes with an independence oracle that returns for all sets $A \subseteq F$ whether $A \in \mathcal{I}$ or not. It shall be noted that for many subclasses of matroids an independence oracle can be implemented in polynomial time.

Another way of representing matroids is via a rank function $\mathrm{rk}: 2^{F} \rightarrow \mathbb{N}$. Every sub-cardinal, monotonic and sub-modular function rk gives rise to a matroid whose independent sets then are defined as $\{A \subseteq F: \operatorname{rk}(A)=|A|\}$. If the independent sets are known a priori via an independence oracle the rank function is defined as $\operatorname{rk}(A)=\max _{I \in \mathcal{I}: I \subseteq A}|I|$. With a slight abuse of notation, we define for a matroid $M=(F, \mathcal{I})$ the rank of the matroid itself as $\operatorname{rk}(M)=\operatorname{rk}(F)$.

To present our positive results for matroid bottleneck congestion games in a general framework we give the definition of matroid union. This concept has been introduced by Nash-Williams [39] and Edmonds [15].

Definition 2 (Matroid union) Let $M_{1}=\left(S_{1}, \mathcal{I}_{1}\right), \ldots, M_{k}=\left(S_{k}, \mathcal{I}_{k}\right)$ be matroids. Define the union of these matroids as $M_{1} \vee \cdots \vee M_{k}=\left(S_{1} \cup \cdots \cup S_{k}, \mathcal{I}_{1} \vee \cdots \vee \mathcal{I}_{k}\right)$ where

$$
\mathcal{I}_{1} \vee \cdots \vee \mathcal{I}_{k}=\left\{I_{1} \cup \cdots \cup I_{k}: I_{1} \in \mathcal{I}_{1}, \ldots, I_{k} \in \mathcal{I}_{k}\right\}
$$

Nash-Williams proved that for $k$ matroids $M_{1}=\left(S_{1}, \mathcal{I}_{1}\right), \ldots, M_{k}=\left(S_{k}, \mathcal{I}_{k}\right)$ their union $M_{1} \vee \cdots \vee M_{k}$ is a matroid again. The maximum cardinality of an independent set in $\mathcal{I}_{1} \vee \cdots \vee \mathcal{I}_{k}$ equals the maximum cardinality of a common independent set of two suitably constructed matroids. This observation reduces the problem of finding a maximum-size set in $\mathcal{I}_{1} \vee \cdots \vee \mathcal{I}_{k}$ to the intersection problem of two matroids, which can be solved in polynomial time, see Cunningham [13].

\section{Appendix B: Description of $G(n)$}

In this section, we recapitulate the construction of $G(n)$ from [45]. This shows that (bottleneck) congestion games do not converge quickly to a PNE even if the players only perform unilateral $\alpha$-improving moves.

We construct a (bottleneck) congestion game $G(n)$ that resembles a recursive run of $n$ programs, i.e., sequences of unilateral $\alpha$-improving moves. After its activation, program $i$ triggers a run of program $i-1$, waits until it finishes its run, and triggers it a second time. These sequences are deterministic apart from the order in which some auxiliary players make their improvement steps.

A program $i$ is implemented by a gadget $G_{i}$ consisting of a main player that we ${\text { call } \text { Main }_{i} \text { and eight auxiliary players called Block }}_{i}^{1}, \ldots$, Block $_{i}^{8}$. The main player has nine strategies numbered from 1 to 9 . Each auxiliary player has two strategies, a first and a second one. A gadget $G_{i}$ is idle if all of its players play their first strategy. Gadget $G_{i+1}$ activates gadget $G_{i}$ by increasing the delay of (the bottleneck resource in) the first strategy of player $\operatorname{Main}_{i}$. In the following sequence of improvement steps the 
Fig. 2 Definition of the strategies of the players Block ${ }_{i}^{j}$

\begin{tabular}{|l|l|l|}
\hline Strategies of Block \\
$i$ & Resources & Delays \\
\hline \hline$(1)$ & $t_{i}^{j}$ & $\delta^{i-1} / 2 \alpha^{2} \delta^{i-1}$ \\
& $b_{i}^{j}$ & $\delta^{i-1} / \delta^{i+2}$ \\
\hline$(2)$ & $c_{i}^{1}$ & $2 \alpha \delta^{i-1} / \delta^{i+2}$ \\
\hline
\end{tabular}

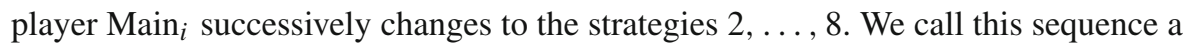
run of $G_{i}$. During each run, Main $_{i}$ activates gadget $G_{i-1}$ twice by increasing the delay of the (bottleneck resource in the) first strategy of Main $_{i-1}$. Gadget $G_{i+1}$ is blocked (by player Block $i_{i}^{8}$ ) until player $\mathrm{Main}_{i}$ reaches its strategy 9. Then $G_{i+1}$ continues its run, that is, it decreases the delay of the bottleneck resource in the first strategy of player $\operatorname{Main}_{i}$, waits until gadget $G_{i}$ becomes idle again, and afterwards triggers a second run of $G_{i}$. The role of the auxiliary players of $G_{i}$ is to control the strategy changes of Main $_{i}$ and Main $i+1$.

In the initial state $s$, every gadget $G_{i}$ with $1 \leq i \leq n-1$ is idle. Gadget $G_{n}$ is activated. In every improvement path starting from $s$, gadget $G_{i}$ is activated $2^{n-i}$ times, which yields the theorem.

Now we go into the details of our construction. The (bottleneck) congestion game $G(n)$ consists of the gadgets $G_{1}, \ldots, G_{n}$. Each gadget $G_{i}$ consists of a player Main $_{i}$ and the players Block ${ }_{i}^{1}, \ldots$, Block $_{i}^{8}$. The nine strategies of a player Main Mre given $_{i}$ in Fig. 3. The two strategies of a player Block ${ }_{i}^{j}$ are given in Fig. $2 . \delta=10 \alpha^{9}$ is a scaling factor for the delay functions.

The auxiliary players implement a locking mechanism. The first strategy of player $\operatorname{Block}_{i}^{j}$ is $\left\{t_{i}^{j}, b_{i}^{j}\right\}$ and its second strategy is $\left\{c_{i}^{j}\right\}$. The delays of the resources $b_{i}^{j}$ and $c_{i}^{j}$ are relatively small ( $\delta^{i-1}$ and $2 \alpha \delta^{i-1}$, respectively) if allocated by only one player. If they are allocated by two or more players, however, then each of them induce a significantly larger delay of $\delta^{i+2}$. Theses resources are also part of the strategies of $\operatorname{Main}_{i}$ or $\operatorname{Main}_{i+1}$. Note, that neither Main nor Main $_{i+1}$ has an incentive to change to a strategy having a delay of $\delta^{i+2}$ or more. The delay of the resource $t_{i}^{j}$ is chosen such that Block ${ }_{i}^{j}$ has an incentive to change to its second strategy if Main $_{i}$ allocates $^{\prime}$ this resource. If $\operatorname{Main}_{i}$ neither allocates this resource nor the resource $b_{i}^{j}$, it has an incentive to change to its first strategy. Due to scaling factor $\delta^{i-1}$ the delays of the resource $t_{i}^{j}$ do not affect the preferences of $\operatorname{Main}_{i}$.

These definitions yield the following properties. If auxiliary player Block ${ }_{i}^{j}$ of gadget $G_{i}$ plays its first strategy then this prevents $\operatorname{Main}_{i}$ from choosing strategy $j+2$. Player Block ${ }_{i}^{j}$ has an incentive to change to its second strategy only if player Main ${ }_{i}$ chooses its strategy $j+1$. By this mechanism, we ensure that $\operatorname{Main}_{i}$ chooses the strategies 1 to 8 in the right order. In addition, the first strategy of Block $_{i}^{8}$ prevents Main $_{i+1}$ from going to strategy 4 or 8 . This ensures that Main $i+1$ waits until the run of player $\operatorname{Main}_{i}$ is completed. Furthermore, Main $i+1$ can enter into strategy 3 or 7 only if all auxiliary players of gadget $G_{i}$ use their first strategy. This ensures that a run starts with all auxiliary players being in their first strategy.

This shows that in every sequence of improvement steps from $s$ to a Nash equilibrium in the (bottleneck) congestion game $G(n)$ each gadget $i$ is activated $2^{n-i}$ times. 
Fig. 3 Definition of the strategies of the players Main $i$. The delay of resource $e_{n}^{1}$ is constantly $9 \alpha^{9} \delta^{n}$

\begin{tabular}{|c|c|c|}
\hline Strategy & Resources & Delays \\
\hline (1) & $\overline{e_{i}^{1}}$ & $\overline{\delta^{i} / 9 \alpha^{9} \delta^{i}}$ \\
\hline (2) & $\begin{array}{l}e_{i}^{2} \\
c_{i-1}^{1}, \ldots, c_{i-1}^{9} \\
t_{i}^{1}\end{array}$ & $\begin{array}{l}8 \alpha^{8} \delta^{i} \\
2 \alpha \delta^{i-2} / \delta^{i+1} \\
\delta^{i-1} / 2 \alpha^{2} \delta^{i-1}\end{array}$ \\
\hline (3) & $\begin{array}{l}e_{i}^{3} \\
e_{i-1}^{1} \\
t_{i}^{2} \\
b_{i}^{1}\end{array}$ & $\begin{array}{l}7 \alpha^{7} \delta^{i} \\
\delta^{i-1} / 9 \alpha^{9} \delta^{i-1} \\
\delta^{i-1} / 2 \alpha^{2} \delta^{i-1} \\
\delta^{i-1} / \delta^{i+2}\end{array}$ \\
\hline (4) & $\begin{array}{l}e_{i}^{4} \\
b_{i-1}^{8} \\
t_{i}^{3} \\
b_{i}^{2}\end{array}$ & $\begin{array}{l}6 \alpha^{6} \delta^{i} \\
\delta^{i-2} / \delta^{i+1} \\
\delta^{i-1} / 2 \alpha^{2} \delta^{i-1} \\
\delta^{i-1} / \delta^{i+2}\end{array}$ \\
\hline (5) & $\begin{array}{l}e_{i}^{5} \\
t_{i}^{4} \\
b_{j}^{3}\end{array}$ & $\begin{array}{l}5 \alpha^{5} \delta^{i} \\
\delta^{i-1} / 2 \alpha^{2} \delta^{i-1} \\
\delta^{i-1} / \delta^{i+2}\end{array}$ \\
\hline (6) & $\begin{array}{l}e_{i}^{\frac{l}{6}} \\
c_{i-1}^{1}, \ldots, c_{i-1}^{9} \\
t_{i}^{5} \\
b_{i}^{4}\end{array}$ & $\begin{array}{l}4 \alpha^{4} \delta^{i} \\
2 \alpha \delta^{i-2} / \delta^{i+1} \\
\delta^{i-1} / 2 \alpha^{2} \delta^{i-1} \\
\delta^{i-1} / \delta^{i+2}\end{array}$ \\
\hline (7) & $\begin{array}{l}e_{i}^{7} \\
e_{i-1}^{1} \\
t_{i}^{0} \\
b_{i}^{5}\end{array}$ & $\begin{array}{l}3 \alpha^{3} \delta^{i} \\
\delta^{i-1} / 9 \alpha^{9} \delta^{i-1} \\
\delta^{i-1} / 2 \alpha^{2} \delta^{i-1} \\
\delta^{i-1} / \delta^{i+2}\end{array}$ \\
\hline (8) & $\begin{array}{l}e_{i}^{8} \\
b_{i-1}^{8} \\
t_{i}^{7} \\
b_{i}^{6}\end{array}$ & $\begin{array}{l}2 \alpha^{2} \delta^{i} \\
\alpha \delta^{i-2} / \delta^{i+1} \\
\delta^{i-1} / 2 \alpha^{2} \delta^{i-1} \\
\delta^{i-1} / \delta^{i+2}\end{array}$ \\
\hline (9) & $\begin{array}{l}e^{9} \\
t_{i}^{8} \\
b_{i}^{7}\end{array}$ & $\begin{array}{l}\alpha \delta^{i} \\
\delta^{i-1} / 2 \alpha^{2} \delta^{i-1} \\
\delta^{i-1} / \delta^{i+2}\end{array}$ \\
\hline
\end{tabular}

One can easily check that every improvement step of a player decreases its delay (of the bottleneck resource) by a factor of at least $\alpha$ and every delay function satisfies the $\beta$-bounded-jump condition with $\beta=\delta^{3}$ with $\delta=10 \alpha^{9}$.

\section{References}

1. Ackermann, H., Berenbrink, P., Fischer, S., Hoefer, M.: Concurrent imitation dynamics in congestion games. In: Proceedings of the 28th Symposium Principles of Distributed Computing (PODC), pp. 63-72 (2009)

2. Ackermann, H., Röglin, H., Vöcking, B.: On the impact of combinatorial structure on congestion games. J. ACM 55(6), 25:1-25:22 (2008)

3. Albers, S.: On the value of coordination in network design. SIAM J. Comput. 38(6), 2273-2302 (2009)

4. Andelman, N., Feldman, M., Mansour, Y.: Strong price of anarchy. Games Econ. Behav. 65(2), 289-317 (2009)

5. Aumann, R.: Acceptable points in general cooperative n-person games. In: Proceedings of the Contributions to the Theory of Games IV, vol. 40 of Annals of Mathematics Study, pp. 287-324. Princeton University Press, Princeton (1959)

6. Banner, R., Orda, A.: Bottleneck routing games in communication networks. IEEE J. Sel. Area Comm. 25(6), 1173-1179 (2007)

7. Bérczi, K., Frank, A.: Packing arborescences. Technical Report TR-2009-04, Egerváry Research Group on Combinatorial Optimization (2009) 
8. Bhalgat, A., Chakraborty, T., Khanna, S.: Nash dynamics in congestion games with similar resources. In: Proceedings of the 5th International Workshop Internet \& Network Economics (WINE), pp. 362-373 (2009)

9. Busch, C., Magdon-Ismail, M.: Atomic routing games on maximum congestion. Theoret. Comput. Sci. 410(36), 3337-3975 (2009)

10. Chakraborty, T., Khanna, S.: Nash dynamics in constant player and bounded jump congestion games. In: Proceedings of the 2nd International Symposium Algorithmic Game Theory (SAGT), pp. 196-207 (2009)

11. Chien, S., Sinclair, A.: Convergence to approximate Nash equilibria in congestion games. Games Econ. Behav. 71(2), 315-327 (2011)

12. Cole, R., Dodis, Y., Roughgarden, T.: Bottleneck links, variable demand, and the tragedy of the commons. In: Proceedings of the 17th Symposium Discrete Algorithms (SODA), pp. 668-677 (2006)

13. Cunningham, W.: Improved bounds for matroid partition and intersection algorithms. SIAM J. Comput. 15(4), 948-957 (1986)

14. de Keijzer, B., Schäfer, G., Telelis, O.: On the inefficiency of equilibria in linear bottleneck congestion games. In: Proceedings of the 3rd International Symposium Algorithmic Game Theory (SAGT), pp. 335-346 (2010)

15. Edmonds, J.: Matroid partition. In: Dantzig, G.B., Veinott, A.F. (eds.) Mathematics of the Decision Sciences, pp. 335-345. AMS, Providence, RI (1968)

16. Epstein, A., Feldman, M., Mansour, Y.: Efficient graph topologies in network routing games. Games Econ. Behav. 66, 115-125 (2009)

17. Epstein, A., Feldman, M., Mansour, Y.: Strong equilibrium in cost sharing connection games. Games Econ. Behav. 67(1), 51-68 (2009)

18. Fabrikant, A., Papadimitriou, C., Talwar, K.: The complexity of pure Nash equilibria. In: Proceedings of the 36th Symposium Theory of Computing (STOC), pp. 604-612 (2004)

19. Feldman, M., Tamir, T.: Approximate strong equilibrium in job scheduling games. J. Artif. Intell. Res. 36, 387-414 (2009)

20. Feldman, M., Tennenholtz, M.: Structured coalitions in resource selection games. ACM Trans. Intell. Syst. Tech. 1(1), 4 (2010)

21. Fiat, A., Kaplan, H., Levy, M., Olonetsky, S.: Strong price of anarchy for machine load balancing. In: Proceedings of the 34th Intl. Coll. Automata, Languages and Programming (ICALP), pp. 583-594 (2007)

22. Fortune, S., Hopcroft, J., Wyllie, J.: The directed subgraph homeomorphism problem. Theoret. Comput. Sci. 10, 111-121 (1980)

23. Gabow, H.: A matroid approach to finding edge connectivity and packing arborescences. J. Comput. Syst. Sci. 50(2), 259-273 (1995)

24. Gairing, M., Monien, B., Tiemann, K.: Routing (un-)splittable flow in games with player-specific linear latency functions. ACM Trans. Algorithms 7(3), 31 (2011)

25. Harks, T., Klimm, M., Möhring, R.: Strong Nash equilibria in games with the lexicographical improvement property. In: Proceedings of the 5th International Workshop Internet \& Network Economics (WINE), pp. 463-470 (2009)

26. Hoefer, M., Penn, M., Polukarov, M., Skopalik, A., Vöcking, B.: Considerate equilibrium. In: Proceedings of the 22nd International Joint Conference Artificial Intelligence (IJCAI), pp. 234-239 (2011)

27. Hoefer, M., Skopalik, A.: On the complexity of Pareto-optimal Nash and strong equilibria. In: Proceedings of the 3rd International Symposium Algorithmic Game Theory (SAGT), pp. 312-322 (2010)

28. Holzman, R., Law-Yone, N.: Strong equilibrium in congestion games. Games Econ. Behav. 21(1-2), 85-101 (1997)

29. Johnson, D., Papadimitriou, C., Yannakakis, M.: How easy is local search? J. Comput. Syst. Sci. 37, 79-100 (1988)

30. Kannan, R., Busch, C.: Bottleneck congestion games with logarithmic price of anarchy. In: Proceedings of the 3rd International Symposium Algorithmic Game Theory (SAGT), pp. 222-233 (2010)

31. Keshav, S.: An Engineering Approach to Computer Networking: ATM Networks, the Internet, and the Telephone Network. Addison-Wesley, Boston, MA (1997)

32. Konishi, H., Breton, M.L., Weber, S.: Equilibria in a model with partial rivalry. J. Econ. Theory 72(1), 225-237 (1997)

33. Korte, B., Vygen, J.: Combinatorial Optimization: Theory and Algorithms. Springer, Berlin (2002) 
34. Leonardi, S., Sankowski, P.: Network formation games with local coalitions. In: Proceedings of the 26th Symposium Principles of Distributed Computing (PODC), pp. 299-305 (2007)

35. Mavronicolas, M., Milchtaich, I., Monien, B., Tiemann, K.: Congestion games with player-specific constants. In: Proceedings of the 32nd International Symposium Mathematical Foundations of Computer Science (MFCS), pp. 633-644 (2007)

36. Mazalov, V., Monien, B., Schoppmann, F., Tiemann, K.: Wardrop equilibria and price of stability for bottleneck games with splittable traffic. In: Proceedings of the 2nd International Workshop Internet \& Network Economics (WINE), pp. 331-342 (2006)

37. Milchtaich, I.: Congestion games with player-specific payoff functions. Games Econ. Behav. 13(1), 111-124 (1996)

38. Monderer, D., Shapley, L.: Potential games. Games Econom. Behav. 14, 1124-1143 (1996)

39. Nash-Williams, C.: An application of matroids to graph theory. In: Rosenstiehl, P. (ed.) Theory of Graphs; Proceedings of an International Symposium in Rome 1966, pp. 263-265 (1967)

40. Qiu, L., Yang, Y.R., Zhang, Y., Shenker, S.: On selfish routing in internet-like environments. IEEE/ACM Trans. Netw. 14(4), 725-738 (2006)

41. Rosenthal, R.: A class of games possessing pure-strategy Nash equilibria. Int. J. Game Theory 2, 65-67 (1973)

42. Roughgarden, T.: Routing games. In: Nisan, N., Tardos, É., Roughgarden, T., Vazirani, V. (eds.) Algorithmic Game Theory Chapter 18, Cambridge University Press, Cambridge (2007)

43. Rozenfeld, O., Tennenholtz, M.: Strong and correlated strong equilibria in monotone congestion games. In: Proceedings of the 2nd International Workshop Internet \& Network Economics (WINE), pp. 74-86 (2006)

44. Schrijver, A.: Combinatorial Optimization: Polyhedra and Efficiency. Springer, Berlin (2003)

45. Skopalik, A., Vöcking, B.: Inapproximability of pure Nash equilibria. In: Proceedings of the 40th Symposium Theory of Computing (STOC), pp. 355-364 (2008)

46. Syrgkanis, V.: Equilibria in congestion game models: Existence, complexity and efficiency. Master's thesis, National Technical University of Athens (2009)

47. Voorneveld, M., Borm, P., van Megen, F., Tijs, S., Facchini, G.: Congestion games and potentials reconsidered. Int. Game Theory Rev. 1(3), 283-299 (1999) 